\title{
The association between ambient air pollution and pulmonary tuberculosis: a systematic review protocol
}

\author{
Christian Akem Dimala ${ }^{1,2,3}$, Benjamin Momo Kadia ${ }^{4^{*}}$ (i] and Anna Hansell ${ }^{2}$
}

\begin{abstract}
Background: There is inconclusive evidence on the association between ambient air pollution and pulmonary tuberculosis-related hospital admission and mortality. This review aims to assess if and to what extent, selected air pollutants are associated to pulmonary tuberculosis (PTB) incidence, hospital admissions and mortality.

Methods: This will be a systematic review and meta-analysis of studies published in English between January 1st, 1946 and October 31st, 2020, quantitatively assessing the association between air pollutants and PTB incidence, hospital admissions and mortality. A comprehensive search strategy will be used to search the databases: Medline, Embase, Scopus and The Cochrane Library. Retrieved studies will be screened based on the set eligibility criteria and data will be extracted from eligible studies. Extracted data will be analysed on STATA version 14.0 software. Studies included will be assessed for their quality using the respective Study Quality Assessment Tools of the National Health Institute. The quality of the evidence on the study outcomes will be graded using the Grading of Recommendations Assessment, Development and Evaluation (GRADE) approach. The risk of bias will be assessed using Cochrane's Risk of Bias In Non-randomized Studies-of Interventions (ROBINS-I) tool. Study characteristics, socio-demographic and clinical characteristics across eligible studies will be summarised and presented. Pooled estimates of the measures of association between air pollutants and PTB incidence, hospital admission and mortality will be obtained through randomeffect meta-analyses models and the respective $I^{2}$ test statistics will be reported. Meta-regression analyses will be done in case of significant between-study heterogeneity.
\end{abstract}

Keywords: Pulmonary tuberculosis, Ambient air pollution, Association

\section{Background}

Significant progress has been made worldwide in the control of the tuberculosis (TB) epidemic over the past two and a half decades [1]. Nevertheless TB still remains a global health emergency, being one of the top 10 causes of death worldwide and the leading cause of death from a single infectious agent [2]. In 2018, up to 10 million new cases of tuberculosis and 1.5 million tuberculosis-related deaths were reported [2]. Despite this significant progress

\footnotetext{
*Correspondence: benjaminmomokadia@gmail.com

${ }^{4}$ Department of Clinical Sciences, Liverpool School of Tropical Medicine, Liverpool, UK
}

Full list of author information is available at the end of the article in TB control over the years, much still needs to be done to end the TB epidemic by the next decade. At the present rate of $2 \%$ annual decrease in tuberculosis incidence worldwide, there are reasonable concerns that the World Health Organisation's (WHO) sustainable development goal (SDG) to end the TB epidemic by 2030 [3] will not be attained globally, most especially with the growing resistance to Rifampicin and the emergence of drug-resistant TB which currently constitutes a public health crisis $[2,4]$. Of more pressing concern is the End TB strategy 2020 milestones of a $35 \%$ reduction in TB deaths and a $20 \%$ reduction in TB incidence rate [2] which are unlikely to be met. This is particularly important as TB remains 
a significant economic burden to affected households, despite being easily treatable and preventable when diagnosed and managed in an appropriate and timely manner. The commonest predisposing factors to TB infection are other health risks such as smoking, diabetes, human immunodeficiency virus (HIV) and social determinants of health such as poverty, malnutrition, poor ventilation and over-crowding among others $[2,5]$. This therefore means that attaining the WHO's SDG target of ending the TB epidemic worldwide by 2030 [2] requires a multifaceted and multi-sectorial approach to TB prevention, case identification, management and control of its health and social determinants $[5,6]$.

Addressing all known TB risk factors from all perspectives is therefore essential in tackling the TB epidemic by the end of the decade. With the increasing global urbanisation, transportation-related emissions and increased energy consumption, air pollution has become a global problem and will be an important factor to deal with in the fight against TB. This is because there are growing concerns of ambient air pollution being linked to increased TB-related hospital admissions and deaths [7, 8]. There is a well-known association between different air pollutants and cardio-respiratory diseases in both adults and children [9-11]. Several large reports have linked air pollutants such as particulate matter (PM), ozone $\left(\mathrm{O}_{3}\right)$ and nitrogen dioxide $\left(\mathrm{NO}_{2}\right)$ among others to both the development and exacerbation of airway disease including asthma and COPD [12-14] and other respiratory conditions $[15,16]$. Air pollutants when inhaled, suppress the natural defence barriers of the respiratory tract by inhibiting macrophage action and muco-ciliary clearance, and initiating a chronic inflammatory response through the generation of inflammatory mediators and free-radicals, thereby increasing susceptibility to infections and sensitivity to allergens [12, 17, 18]. PTB has been previously shown to be associated with indoor pollution from activities such as smoking, biomass fuel burning [19-21]. Despite this background knowledge of the association between indoor air pollution and PTB risk, not much has been reported about the contribution of ambient air pollution to the risk of PTB development or exacerbation. Rajaei et al. using geographical information systems, showed a possible association between long-term exposure to $\mathrm{PM}_{2.5}$ and $\mathrm{CO}$ and $\mathrm{TB}$ development but not with $\mathrm{SO}_{2}, \mathrm{NO}$, and $\mathrm{PM}_{10}$ [7]. Another study reported a potential link between $\mathrm{PM}_{2.5}, \mathrm{PM}_{10}, \mathrm{SO}_{2}, \mathrm{NO}_{2}$ and active PTB [8]. On the other hand Hwang et al. found no link between $\mathrm{PM}_{10}, \mathrm{CO}, \mathrm{NO}_{2}, \mathrm{O}_{3}$ and $\mathrm{PTB}$, but rather with $\mathrm{SO}_{2}$ [22]. Variations in study settings, study designs and study populations could potentially account for these contrasting findings across studies. The systematic review conducted by Popovic et al. in 2018 which is the only published synthesis of studies on the link between ambient pollution and PTB to date showed mixed results [23]. This review reported that $\mathrm{PM}_{2.5}$ was the air pollutant most frequently associated with PTB. This review also found no evidence of association between $\mathrm{CO}$ and $\mathrm{O}_{3}$ and active PTB and limited evidence of associations between $\mathrm{PM}_{10}, \mathrm{SO}_{2}$, and $\mathrm{NO}_{2}$ and active PTB [23]. The systematic review, however, did not quantitatively assess to what extent these air pollutants might be associated to active PTB. Also, several other studies which provide more evidence on this topic were published after the systematic review. The uncertainty of which air pollutants could be linked to PTB and to what extent, is therefore amenable to further exploration.

This review will enable us to ascertain if available evidence suggests ambient air pollution is associated to PTB and to what extent these air pollutants are linked to PTB incidence, tuberculosis-related hospital admissions and deaths, by systematically reviewing and quantitatively synthesising published evidence on this topic. This is particularly relevant as identifying potentially modifiable environmental determinants of PTB and the socio-demographic and clinical characteristics of at-risk population groups would greatly help to inform policy and decision making with regards to targeted control measures and prevention strategies, in line with WHO's SDG goal to end the TB epidemic in 2030. [2,3].

\section{Objectives of the review}

This review has as primary review questions: Is ambient air pollution associated with PTB incidence, mortality and hospital admissions? And if so, what air pollutants are involved? The specific objectives of the review are:

1. To determine if there is an association between air pollution and PTB incidence, mortality and hospital admissions

2. To determine to what extent selected air pollutants $\left(\mathrm{PM}_{2.5}, \mathrm{PM}_{10}, \mathrm{NO}_{2}, \mathrm{SO}_{2}, \mathrm{CO}, \mathrm{O}_{3}\right)$ are associated to PTB incidence, mortality and/or hospital admissions

\section{Methods}

The study has been registered with the international prospective register of systematic reviews (PROSPERO registration number: CRD42020165888). The RepOrting standards for Systematic Evidence Syntheses (ROSES) for systematic review protocols [24] were used to report this protocol as presented in Additional file 1. Likewise, the completed systematic review and meta-analysis will be reported in accordance with the ROSES for systematic review reports. Amendments made to this protocol will be documented and justified accordingly. 


\section{Searching for articles}

This will be a systematic review of studies that quantitatively test for any association between ambient air pollution and PTB including drug-resistant TB. The search strategy to be used is presented in Table 1 . The search will be run by two independent reviewers (CAD and BMK). The medical subject headings (MeSH) searches of the keywords; air pollution; tuberculosis, incidence, mortality, hospital admission and hospitalisation will be combined with the respective free-text searches of these keywords and more keywords using the Boolean operator 'OR'. The respective outputs from these initial searches will then be combined using the Boolean operator 'AND' to obtain the definitive search results. The databases to be searched will include Medline, Embase, Scopus and The Cochrane Library and all searches will be conducted in English. The reference list of eligible articles will be searched, but no other specialist searches will be done including grey literature search, stakeholder contacts, organisational website searches or public calls for literature.

\section{Article screening and eligibility criteria Screening process}

Articles returned by the search will be saved on Zotero Version 5.0 reference management software from where duplicates of articles will be removed by the principal investigator (CAD). The titles and abstracts of the remaining articles will then be screened by two independent reviewers (CAD and BMK) to identify and exclude studies which are out of scope and unrelated to the study objectives. For consistency checks, the two reviewers will then check with each other the number of records they each retained for full text review to make sure their numbers match and any discrepancies will be reviewed and addressed by both reviewers. The reviewers will then review the full texts of the retained articles for their eligibility based on the study selection criteria. Thereafter, the two reviewers will compare their final eligible list of studies to ensure concordance in their selection. In case of any mismatch in the eligible studies identified by both reviewers, these two reviewers will again review the discrepancy to agree on a final list. Any disagreement between the two reviewers at any point during the article screening process will be subject to arbitration by a third reviewer (AH). More studies will be assessed for eligibility by reviewing the reference list of eligible studies. A list of studies excluded following full text review will be made with the respective reasons for their exclusion.

\section{Eligibility criteria}

The following studies will be included:

1. Studies published in English between January 1st, 1946 and the date of the search

2. Population: Studies focused on patients of all ages with confirmed PTB including drug-resistant TB

3. Exposure: Studies that report on any of the air pollutants; carbon monoxide (CO), nitrogen dioxide $\left(\mathrm{NO}_{2}\right)$, sulphur dioxide $\left(\mathrm{SO}_{2}\right)$, ozone $\left(\mathrm{O}_{3}\right)$, particulate matter $\leq 2.5$ micrometres $\left(\mathrm{PM}_{2.5}\right)$ and/or particulate matter $\leq 10$ micrometres $\left(\mathrm{PM}_{10}\right)$

4. Comparator: more or less air pollution

5. Outcomes: Studies that report risk of TB incidence, hospital admission and/or mortality from PTB or some other measure of effect from which the risk or odds ratios can be calculated

6. Study design/Other: Cross-sectional, case-control, cohorts, case-crossover and time-series studies that report on the association between ambient air pollution and PTB.

The following studies will be excluded

1. Population: Studies that report on patients of all ages with respiratory diseases other than PTB and studies that report on extrapulmonary TB rather than PTB

Table 1 Ovid Medline and Embase Search Strategy for study on the association between ambient air pollution and pulmonary tuberculosis

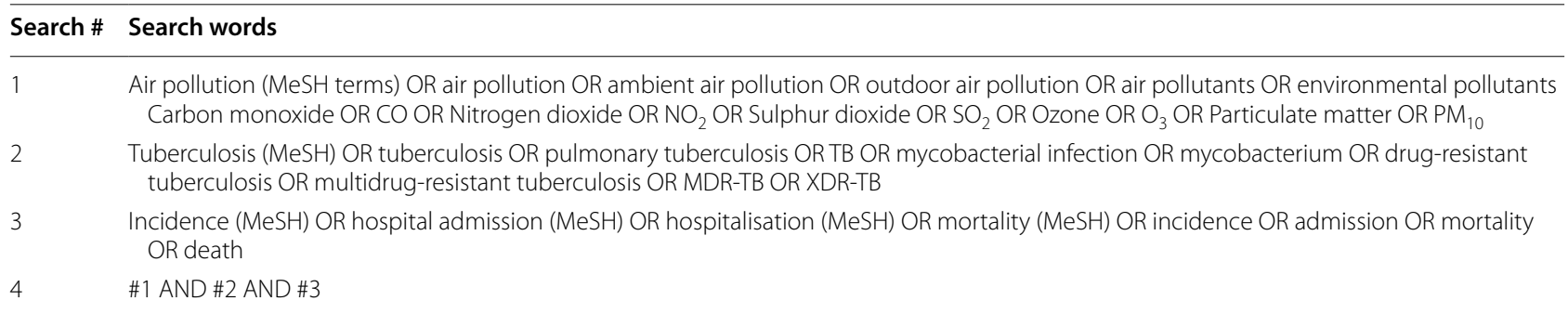


2. Exposure: Studies reporting exclusively on indoor air pollution with no assessment of ambient or outdoor air pollution

3. Outcomes: Studies reporting outcomes related to PTB in combination with other respiratory diseases. Studies from which data on measures of effect/association such as relative risk, risk ratios and odds ratios are inadequately reported

4. Study design/Other: Conference abstracts, editorials, letters, opinion papers, same studies published in different journals with the same or a different title

For prospective studies with multiple publication of their results over time, only the most recent publication that provides adequate quantitative data will be considered for the meta-analysis. Data from single-pollutant models will be given preference over data from multipollutant models for studies that provide both. Data from multi-pollutant models will be considered if there is consistency in the air-pollutants considered in the multipollutant models across two or more studies. Adjusted measures of effect/association will be given preference over crude measures when both are reported.

\section{Study validity assessment}

Assessment of study quality and risk of bias will be done by two independent reviewers (CAD and BMK). Particular attention will be paid to; the study designs; the availability of clearly reported measures of effect/association on the outcomes of interest; and the reporting and control of confounders. The quality of studies will be graded as poor, fair, good using appropriate Study Quality Assessment Tools of the National Health Institute/National Heart, Lung and Blood Institute (NHI/ NHLBI) [25]. The criteria used by the quality assessment tool assess the method of randomisation of the participants to the study, treatment concealment, blinding of participants and investigators, the similar across groups at baseline, the drop-out rate, the extent of adherence of participants to the intervention protocol and the reported sample sizes. The study quality will be considered during study synthesis to assess its effect on the overall results. The risk of bias will be assessed using Cochrane's Risk Of Bias In Non-randomized Studies-of Interventions (ROBINS-I) tool [27].

The quality of the cumulative evidence on the outcome measures (hospital admissions and mortality) will be assessed and graded as high, moderate, low and very low, using the Grading of Recommendations Assessment, Development and Evaluation (GRADE) approach on the bases of the study limitations, inconsistencies, indirectness, imprecision and publication bias [26].

\section{Data coding and extraction strategy}

Meta-data (first author and publication year), and data on study methods and outcomes of interest will be extracted by the principal investigator (CAD) from the eligible studies, including: Study location (region, country and city or province or county), study design, socio-demographic and clinical characteristics of study participants, study duration, data on pollutants of interest $\left(\mathrm{CO}, \mathrm{NO}_{2}\right.$, $\mathrm{SO}_{2}, \mathrm{O}_{3}, \mathrm{PM}_{2.5}$ and $\mathrm{PM}_{10}$ ), the average ambient temperature over the study period, incidence of PTB during study duration, measures of effect/association (risk ratios, odds ratios, hazard ratio, relative risk, percentage change, and their respective confidence intervals) of unit increments (in $\mu \mathrm{g} / \mathrm{m}^{3}$ ) in the various air pollutant concentrations on the incidence, mortality and hospital admissions due to PTB and confounders reported by the respective studies. Air pollutant data collected will include the method of measurement of air pollutants' concentration and the median and/or mean pollutant concentrations during the study period. Air pollutant concentration units of measurement considered will be $\mu \mathrm{g} / \mathrm{m}^{3}$, parts per million ( $\mathrm{ppm})$, and parts per billion ( $\mathrm{ppb})$. Where different units of air pollutant concentrations are reported, appropriate conversions will be done according to standards to ensure consistency. Where studies provide sufficient data, the appropriate measures of effect and association will be calculated accordingly and added into the data extraction sheet. Potential effect modifiers and confounder such as study location, temperature and seasonality will also be recorded. Relevant missing and unclear data will be sought by directly contacting the authors of the respective studies by their provided emails and will be added to the data extraction sheet once provided. All data extracted and the coding will be double-checked for errors by a second investigator (BMK) and discrepancies in entries will be settled by discussions between both investigators. Disagreements between the investigators will be subject to arbitration by a third investigator (AH). Data will be extracted unto a Microsoft excel office 365 sheet and exported to STATA version 14 statistical software for analysis. Extracted data will be made available as Additional file 1.

\section{Data synthesis and presentation}

A narrative synthesis of the study characteristics (study location, study design, study duration and total participants) will be presented and this will be complemented by figures and tables summarising descriptive statistics such as a means, and frequencies of the socio-demographic and clinical characteristics of the participants in the various studies. Meta-analyses will be done using random-effects models to account for the possibility of 
between-study heterogeneity. Studies with incomplete or missing data and studies from which appropriate measures of effect/association (risk ratios, odds ratio, hazard ratios, relative risk and percentage change) cannot be derived, will not be included in the meta-analysis. The first and second study objectives will be addressed by deriving random-effect pooled estimates for the measures of association between the various air pollutants and the outcomes of interests (hospital admission and mortality) gathered from the various studies. Heterogeneity between studies will be assessed using the Cochrane's Q test and the $\mathrm{I}^{2}$ test statistic reported as a measure of the extent of this heterogeneity. Meta-analysis findings will be summarised in tables and forest plots. Studies assessing exposure to air pollutants at different levels (individual versus population-level) will be analysed separately. Likewise, studies with different methods of measuring air pollutant concentrations will be analysed separately. If data collected allows for the possibility of combining the meta-analysis for studies that used different methods of measurement of air pollutant concentrations, then sub-groups analyses according to the various methods of measurement of air pollutant concentration in the atmosphere will also be done to assess their impact on the overall results. Meta-regression analyses will also be done to assess for between-study heterogeneity and explore other parameters that could affect PTB distribution such as study year, duration and location. Graphical and Statistical assessments of publication bias and small study effect will be conducted by ways of funnel plots and Harbord's and Peter's statistical tests respectively [28]. Meta-regression analyses will be done to explore potential factors that could account for between-study heterogeneity, if any.

The RepOrting standards for Systematic Evidence Syntheses (ROSES) for systematic review protocols [28] were used to report this protocol as presented in Additional file 1 . Likewise, the systematic review and meta-analysis proper once completed will be reported in accordance with the ROSES for systematic review reports. Amendments made to this protocol will be documented and justified accordingly. The findings of this study will be published in a peer-reviewed journal and made widely available to the respective health institutions and health care providers and patients.

We envisage some limitations to this review. The review may overestimate the effect of the various air pollutants and their contribution to the development of PTB. This is because the nature of clinical presentation of the cases admitted to hospital and the synergistic effect of several pollutants makes it clinically impracticable to quantify with precision the impact of the individual air pollutants. Preferentially selecting data from single-pollutant models and including multi-pollutant models including the same air pollutants will help minimise this confounding effect. Also, this study does not take into consideration the contribution of household/indoor air pollution to the increased risk of hospital admission and mortality from PTB, as well as the contribution of other comorbidities or health conditions. Finally, as with all ecological studies, this study will be limited by the ecological fallacy which means individual level exposures to air pollutants cannot accurately be predicted from measurements at city or country level and consequently, associations we may observe after aggregating data from cities or countries may not necessarily hold at the individual level in these populations.

\section{Supplementary information}

Supplementary information accompanies this paper at https://doi. org/10.1186/s13750-020-00213-9.

Additional file 1. The RepOrting standards for Systematic Evidence Syntheses (ROSES) for systematic review protocols for the systematic review protocol on the association between ambient air pollution and pulmonary tuberculosis.

\section{Acknowledgements}

Not applicable.

\section{Authors' contributions}

$\mathrm{CAD}$ and $\mathrm{AH}$ conceived and designed the experiments. CAD produced the manuscript. BMK and $\mathrm{AH}$ reviewed the manuscript. All authors read and approved the final manuscript.

\section{Funding}

None.

Availability of data and materials

Not applicable.

\section{Ethics approval and consent to participate}

This systematic review and meta-analysis does not require ethical approval as it entails a synthesis of data collected from several primary studies. No primary data collection from patients will be done for this systematic review.

\section{Consent for publication}

Not applicable.

\section{Competing interests}

The authors declare that they have no competing interests.

\section{Author details}

${ }^{1}$ Health and Human Development (2HD) Research Network, Douala, Cameroon. ${ }^{2}$ Centre for Environmental Health and Sustainability, University of Leicester, Leicester, UK. ${ }^{3}$ Infectious Diseases Unit, University Hospitals of Leicester NHS Trust, Leicester, UK. ${ }^{4}$ Department of Clinical Sciences, Liverpool School of Tropical Medicine, Liverpool, UK.

Received: 25 August 2020 Accepted: 17 November 2020

Published online: 25 November 2020 


\section{References}

1. GBD Tuberculosis Collaborators. Global, regional, and national burden of tuberculosis, 1990-2016: results from the Global Burden of Diseases, Injuries, and Risk Factors 2016 Study. Lancet Infect Dis. 2018;18(12):1329-49.

2. World Health Organization. Global Tuberculosis Report 2019. Geneva: World Health Organization; 2019.

3. United Nations. Sustainable Development Goals. Goal 3: Ensure healthy lives and promote well-being for all at all ages. https://www.un.org/susta inabledevelopment/health/. Accessed 17 Jan 2020.

4. United Nations General Assembly. Resolution 73/3: Political declaration of the high-level meeting of the General Assembly on the fight against tuberculosis. United Nations; 2018. https://www.un.org/en/ga/search/ view_doc.asp?symbol=A/RES/73/3.

5. Hargreaves JR, Boccia D, Evans CA, Adato M, Petticrew M, Porter JDH. The social determinants of tuberculosis: from evidence to action. Am J Public Health. 2011;101(4):654-62.

6. Grobusch MP, Kapata N. Global burden of tuberculosis: where we are and what to do. Lancet Infect Dis. 2018:18(12):1291-3.

7. Rajaei E, Hadadi M, Madadi M, Aghajani J, Ahmad MM, Farnia P, et al. Outdoor air pollution affects tuberculosis development based on geographical information system modeling. Biomed Biotechnol Res J BBRJ. 2018:2(1):39.

8. Li Z, Mao X, Liu Q, Song H, Ji Y, Xu D, et al. Long-term effect of exposure to ambient air pollution on the risk of active tuberculosis. Int J Infect Dis. 2019;1(87):177-84.

9. Requia WJ, Adams MD, Arain A, Papatheodorou S, Koutrakis P, Mahmoud M. Global association of air pollution and cardiorespiratory diseases: a systematic review, meta-analysis, and investigation of modifier variables. Am J Public Health. 2017;108(S2):S123-30.

10. Atkinson RW, Kang S, Anderson HR, Mills IC, Walton HA. Epidemiological time series studies of PM25 and daily mortality and hospital admissions: a systematic review and meta-analysis. Thorax. 2014;69(7):660-5.

11. Manan N, NoorAizuddin A, Hod R. Effect of air pollution and hospital admission: a systematic review. Ann Glob Health. 2018;84(4):670-8.

12. Kelly FJ, Fussell JC. Air pollution and airway disease. Clin Exp Allergy J Br Soc Allergy Clin Immunol. 2011:41(8):1059-71.

13. Moore E, Chatzidiakou L, Kuku M-O, Jones RL, Smeeth L, Beevers S, et al. Global associations between air pollutants and chronic obstructive pulmonary disease hospitalizations: a systematic review. Ann Am Thorac Soc. 2016:13(10):1814-27.

14. Song Q, Christiani DC, Xiaorong W, Ren J. The global contribution of outdoor air pollution to the incidence, prevalence, mortality and hospital admission for chronic obstructive pulmonary disease: a systematic review and meta-analysis. Int J Environ Res Public Health. 2014;11(11):11822-32.

15. Schwartz J. PM10 ozone, and hospital admissions for the elderly in minneapolis-St. Paul, Minnesota. Arch Environ Health Int J. 1994:49(5):366-74
16. Dockery DW, Speizer FE, Stram DO, Ware JH, Spengler JD, Ferris BG. Effects of inhalable particles on respiratory health of children. Am Rev Respir Dis. 1989;139(3):587-94

17. Gasser M, Riediker M, Mueller L, Perrenoud A, Blank F, Gehr P, et al. Toxic effects of brake wear particles on epithelial lung cells in vitro. Part Fibre Toxicol. 2009;6(1):30.

18. Behndig AF, Mudway IS, Brown JL, Stenfors N, Helleday R, Duggan ST, et al. Airway antioxidant and inflammatory responses to diesel exhaust exposure in healthy humans. Eur Respir J. 2006;27(2):359-65.

19. Sumpter C, Chandramohan D. Systematic review and meta-analysis of the associations between indoor air pollution and tuberculosis. Trop Med Int Health TM IH. 2013;18(1):101-8.

20. Kolappan C, Subramani R. Association between biomass fuel and pulmonary tuberculosis: a nested case-control study. Thorax. 2009;64(8):705-8.

21. Kurmi OP, Sadhra CS, Ayres JG, Sadhra SS. Tuberculosis risk from exposure to solid fuel smoke: a systematic review and meta-analysis. J Epidemiol Community Health. 2014;68(12):1112-8.

22. Hwang S, Kang S, Lee J-Y, Lee JS, Kim HJ, Han SK, et al. Impact of outdoor air pollution on the incidence of tuberculosis in the Seoul metropolitan area, South Korea. Korean J Intern Med. 2014;29(2):183-90.

23. Popovic I, Magalhaes RJS, Ge E, Marks GB, Dong G-H, Wei X, et al. A systematic literature review and critical appraisal of epidemiological studies on outdoor air pollution and tuberculosis outcomes. Environ Res. 2019;170:33-45.

24. Haddaway NR, Macura B, Whaley P, Pullin AS. ROSES RepOrting standards for Systematic Evidence Syntheses: pro forma, flow-diagram and descriptive summary of the plan and conduct of environmental systematic reviews and systematic maps. Environ Evid. 2018;7(1):7.

25. Study Quality Assessment Tools-NHLBI, NIH. https://www.nhlbi.nih.gov/ health-pro/guidelines/in-develop/cardiovascular-risk-reduction/tools. Accessed 2 Nov 2017.

26. What is GRADE? - BMJ Best Practice. https://bestpractice.bmj.com/info/ toolkit/learn-ebm/what-is-grade/. Accessed 17 Jan 2020.

27. Sterne JA, Hernán MA, Reeves BC, Savović J, Berkman ND, Viswanathan M, et al. ROBINS-1: a tool for assessing risk of bias in non-randomised studies of interventions. BMJ. 2016. https://www.bmj.com/content/355/bmj. i4919. Accessed 2 Nov 2020.

28. Sterne JA, Egger M, Moher D. Addressing reporting biases. in: cochrane handbook for systematic reviews of interventions. New York: Wiley; 2008. p. 297-333. https://onlinelibrary.wiley.com/doi/abs/10.1002/9780470712 184.ch10. Accessed 15 Jan 2020

\section{Publisher's Note}

Springer Nature remains neutral with regard to jurisdictional claims in published maps and institutional affiliations.
Ready to submit your research? Choose BMC and benefit from:

- fast, convenient online submission

- thorough peer review by experienced researchers in your field

- rapid publication on acceptance

- support for research data, including large and complex data types

- gold Open Access which fosters wider collaboration and increased citations

- maximum visibility for your research: over 100M website views per year

At BMC, research is always in progress.

Learn more biomedcentral.com/submissions 